Interner Bericht

DESY T-96-03

Juli 1996

\section{Teilchenphysik: \\ Status und Perspektiven}

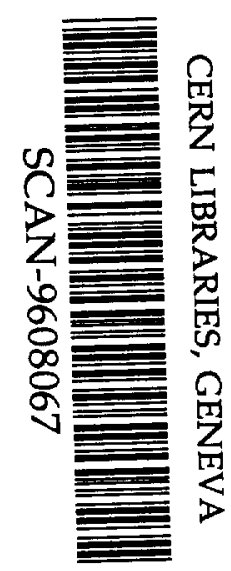

sung 634

von

P. M. Zerwas 
DESY behält sich alle Rechte für den Fall der Schutzrechtserteilung und für die wirtschaftliche Verwertung der in diesem Bericht enthaltenen Informationen vor.

DESY reserves all rights for commercial use of information included in this report, especially in case of filing application for or grant of patents.

"Die Verantwortung für den Inhalt dieses

Internen Berichtes liegt ausschließlich beim Verfasser" 


\section{TEILCHENPHYSIK: STATUS UND PERSPEKTIVEN}

*

P.M. Zerwas

Deutsches Elektronen-Synchrotron DESY, D-22603 Hamburg

*Vortrag anläßlich der 125 Jahrfeier der RWTH Aachen am 26. Oktober 1995 


\section{Einführung}

Eines der tiefgründigsten Prinzipien der Naturphilosopie ist das Reduktionsprinzip, das in seiner Essenz von Thales von Milet und Demokrit eingeführt wurde. In moderner Sprache formuliert besagt es, daß die Vielfalt der makroskopischen Materie eine Folge komplexer Zusammensetzung aus wenigen einfachen Konstituenten ist, zwischen denen eine kleine Zahl fundamentaler Kräfte wirkt.

In dieser hierarchischen Ordnung wird die makroskopische Physik auf den Atomismus zurückgeführt. Die Atome ihrerseits bestehen aus Kernen, umgeben von einer Hülle, die aus Elektronen gebildet wird. Die Kerne sind ebenfalls zusammengesetzte Gebilde und werden von Protonen und Neutronen aufgebaut. Durch Mikroskopierung von Protonen und Neutronen konnte experimentell gezeigt werden, da $ß$ diese Teilchen aus Quarks aufgebaut sind. Abbildung 1 dokumentiert diese Hierarchie der Materieteilchen.

Quarks und Leptonen, die Klasse der elektron-artigen Teilchen, bilden bei der zur Zeit experimentell erreichten Aufösung die innerste Schale der Materie. Zwischen diesen Teilchen wirken vier fundamentale Kräfte: die starke, die elektromagnetische, die schwache Kraft und die Gravitationskraft. Diese einfache Struktur der Materie wird manifest bei Distanzen von $10^{-15} \mathrm{~cm}$. Sie wird vom Standardmodell der Teilchenphysik beschrieben. Neben den Materieteilchen und Kräften umfaßt das Modell den Higgs-Mechanismus als dritte Komponente: die Massen der fundamentalen Teilchen werden durch die Wechselwirkung mit einem skalaren Feld erzeugt. Das Standardmodell der Teilchenphysik bildet die mikroskopische Grundlage der physikalischen Welt. Alle makroskopischen Phänomene lassen sich also letztlich auf die in diesem Modell formulierten Gesetze reduzieren.

Viele Fazetten in der Struktur der Materieteilchen und Kräfte sind experimentell bereits mit sehr hoher Genauigkeit etabliert worden. Für andere Komponenten des Modells, insbesonders die Massen-Erzeugung auf der Basis des HiggsMechanismus, gibt es erste experimentelle, wenn auch nur indirekte Hinweise.

Obwohl das Standardmodell extrem erfolgreich in der Beschreibung der Naturphänomene im Mikrokosmos ist, so kann es trotzdem nicht die ultima ratio der materiellen Welt sein. Es ist zwar gelungen, im Rahmen dieses Modells die elektromagnetischen und schwachen Kräfte zu einer elektroschwachen Wechselwirkung zu vereinheitlichen, jedoch nicht die starke Kraft. Darüber hinaus wird die Gravitationskraft ad hoc als klassisches Phänomen beigefügt, ohne quantentheoretisch inkorporiert zu werden. Dieser Schritt muß jedoch vollzogen werden, wenn bei Abständen im Bereich der Planck-Länge von $10^{-33} \mathrm{~cm}$, einer Energie von $10^{19} \mathrm{GeV}$ entsprechend, die Gravitationswechselwirkung stark wird.

Es gibt im Prinzip vielerlei Möglichkeiten, diese Probleme zu lösen. Ein naheliegender Weg könnte darin bestehen, Quarks, Leptonen und die den Kraftfeldern zugeordneten Teilchen in Sub-Konstituenten bei Abständen unterhalb von $10^{-17} \mathrm{~cm}$ aufzulösen. Es ist jedoch bisher nicht gelungen, ein dynamisches Konzept zu entwickeln, in dessen Rahmen Sub-Konstituenten zu leichten Quarks und Leptonen 


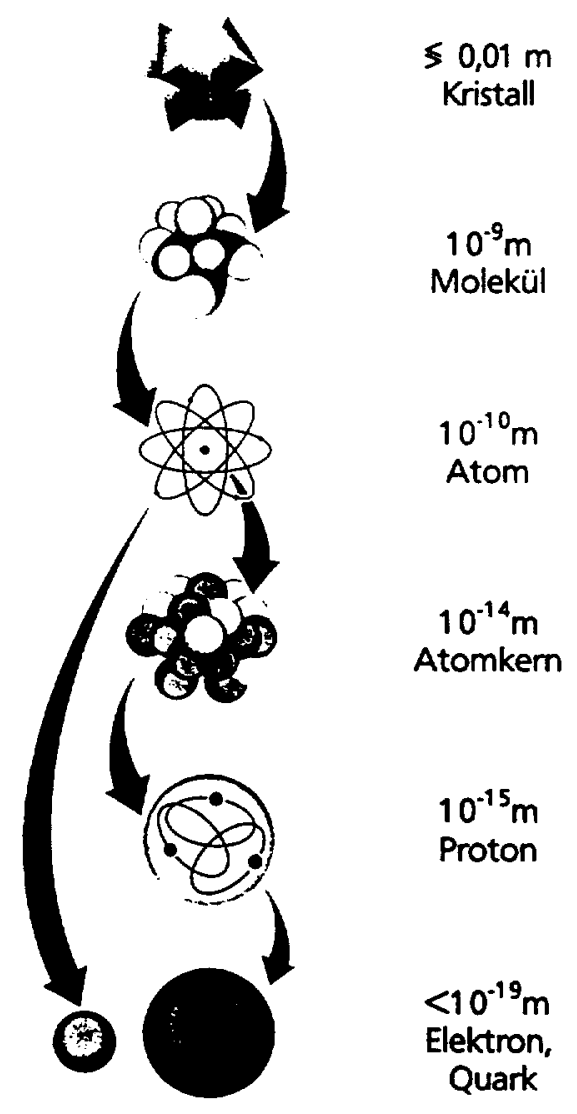

Abbildung 1: Hierarchie der Materieteilchen: Entwicklung von der makroskopischen Physik zum Standardmodell der Teilchenphysik.

mit sehr kleinen Radien zusammengefügt werden konnten - eine Schwierigkeit, die in ihrer Essenz mit dem Unschärfeprinzip der Quantenmechanik verknüpft ist.

Das alternative Konzept basiert auf der Hypothese, daß die Lücke von der heutigen Energieskala im Bereich von $10^{3} \mathrm{GeV}$ bis zur Planck-Skala im Bereich von $10^{19} \mathrm{GeV}$ überbrückt werden kann, ohne daß in einer Folge von jeweils wenigen Größenordnungen der Energie eine Sequenz von neuen fundamentalen Materieschalen existiert. Die Lücke kann in der Tat überbrückt werden, falls im Niederenergiebereich von $10^{2}$ bis $10^{3} \mathrm{GeV}$ die Teilchen des Standardmodells in supersymmetrischen Multipletts gepaart werden, die zur selben Zeit bosonische und fermionische Komponenten enthalten. In einer solchen Theorie können die starke und die elektroschwache Kraft bei einer Energieskala von $10^{16} \mathrm{GeV}$ zu einer einheitlichen Kraft konsistent vereinigt werden. Gestützt, jedoch nicht bewiesen, wird diese zunächst gewagt anmutende Hypothese durch experimentelle Fakten. Das überzeugendste Argument kann aus der sehr genauen Vorhersage dieser Theorie für das Verhältnis der Kopplungen der schwachen und elektromagnetischen Wechselwirkung abgeleitet werden. Die Gravitationswechselwirkung kann im Rahmen einer solchen lokalen Quantenfeldtheorie noch nicht konsistent inkorporiert werden. Die Lösung dieses Problems 
könnte jedoch in Stringtheorien möglich sein, in denen nicht punktförmige Teilchen, sondern linear ausgedehnte Gebilde das physikalische Grundsubstrat bilden.

Es gibt also überzeugende Gründe dafür anzunehmen, daß die Formulierung einer Theorie der Materie, die alle Teilchen und Wechselwirkungsarten vereinheitlicht einschließt, möglich sein wird. Entscheidende experimentelle Meilensteine auf diesem Weg können von der gegenwärtigen und der nächsten Generation von Beschleunigern, dem Protonencollider LHC und zukünftigen $e^{+} e^{-}$Linearcollidern gesetzt werden.

\section{Das Standardmodell der Teilchenphysik}

Das Standardmodell der Teilchenphysik $[1,2]$ besteht aus drei Komponenten:

- Die Materieteilchen werden von Leptonen und Quarks gebildet;

- Die fundamentalen Kräfte werden von Eichfeldern aufgebaut;

- Die Massen der fundamentalen Teilchen werden durch ihre Wechselwirkung mit dem Higgs-Feld erzeugt.

\subsection{Materieteilchen}

Die Materieteilchen, Leptonen und Quarks, ordnen sich in drei Familien mit identischer Symmetriestruktur.

\begin{tabular}{||c|c|c||}
\hline$\nu_{e}$ & $\nu_{\mu}$ & $\nu_{\tau}$ \\
$e^{-}$ & $\mu^{-}$ & $\tau^{-}$ \\
& & \\
$u$ & $c$ & $t$ \\
$d$ & $s$ & $b$ \\
\hline
\end{tabular}

Tabelle 1: Leptonen und Quarks.

Die erste Familie setzt sich aus Elektronen und Neutrinos, sowie up- und down-Quarks zusammen. Neutrinos sind die elektrisch neutralen Partner der Elektronen, die im $\beta$-Zerfall von Kernen entdeckt worden sind. Up- und down-Quarks bauen Protonen und Neutronen auf, welche die Kernmaterie bilden. Die Teilchen in diesen drei Familien sind während der letzten Jahrzehnte experimentell direkt nachgewiesen worden - mit Ausnahme des $\tau$-Neutrinos, für dessen Existenz die in- 
direkte experimentelle Evidenz jedoch überwältigend ist (spätestens der LHC wird diese Scheinlücke in klassischer Weise schließen können).

Auf der gegenwärtigen Skala der experimentellen Auflösung erscheinen Quarks und Leptonen als strukturlose, punktförmige Teilchen. Sie erfüllen damit das Kriterium der Einfachheit im Kontext des Reduktionsprinzips. In Rutherfordähnlichen Experimenten der Elektron-Positron, der Elektron-Quark und der QuarkQuark Streuung sind obere Schranken an die Radien von Leptonen und Quarks bei LEP, HERA und dem Tevatron bestimmt worden: die Radien dieser Teilchen müssen unterhalb von $10^{-17} \mathrm{~cm}[3]$ liegen.

Aus $Z$-Zerfällen ist bei LEP [4] die Anzahl von Neutrinos mit vernachlässigbarer Masse bestimmt worden: $N_{\nu}=2.988 \pm 0.023$. Es kann also nur drei Familien mit der Struktur des Standardmodells im Materie-Sektor geben.

Die Massen der Leptonen und Quarks umfassen einen sehr weiten Bereich. Neutrinomassen müssen, falls sie nicht null sind, im eV Bereich und weit darunter liegen. Geladene Leptonmassen erstrecken sich vom Wert $0.5 \mathrm{MeV}$ der Elektronmasse bis $1.8 \mathrm{GeV}$ der $\tau$-Masse. Quarks besitzen Massen zwischen dem MeV Bereich der $u$ und $d$ Quarks und dem Wert von $175 \mathrm{GeV}$ des top-Quarks, dem schwersten Materieteilchen des Standardmodells. Abgesehen von der klaren Abtrennung der Neutrino-Massen und dem Anwachsen der Massen mit der Familiennummer ist das Muster der geladenen Lepton- und Quark-Massen erratisch. Eine unterliegende Symmetriestruktur in Analogie zu einer Balmer-Serie ist nicht erkennbar, so daß wohl dynamische Wechselwirkungs-Effekte für die spezifischen Massenwerte verantwortlich sein werden.

Neben einer Reihe interessanter Teilfragen gibt es drei herausragende Probleme im Materie-Sektor, die gelöst werden müssen.

\section{(a) TOP-QUARK}

Das sechste Quark im Standardmodell, das top-Quark, ist vor kurzer Zeit am Tevatron im Fermi-Laboratorium direkt nachgewiesen worden. An seiner Existenz hat schon lange kein Zweifel mehr bestanden. Die Messung der Quantenzahlen des $b$-Quarks zeigte, daß ein Iso-Partner mit den charakteristischen Quantenzahlen des top-Quarks existieren mußte. Quantenfluktuationen, die virtuelle top-Quarks involvieren, beeinflussen die Zerfalls- und Produktionseigenschaften des $Z$-Bosons am LEP-Speicherring. Präzisionsmessungen dieser Observablen [4] wurden daher genutzt, die Masse des top-Quarks indirekt zu bestimmen: $m_{t}=180 \pm 14 \mathrm{GeV}$.

In den Proton-Antiproton Kollisionen des Tevatrons werden top-Quarkpaare durch Annihilation leichter Quarks erzeugt. Die direkte Messung der top-Masse [5], $m_{t}=176 \pm 9 \mathrm{GeV}$, ist in sehr guter Übereinstimmung mit der LEP-Analyse - ein Triumph der Quantenfeldtheorie, in der das Standardmodell formuliert ist.

Die Eigenschaften des top-Quarks werden am Tevatron und später am Protonencollider LHC im CERN genauer bestimmt werden. Zu einer wesentlichen Verbesserung dieser Analysen wird jedoch erst die Produktion von top-Quarkpaaren 


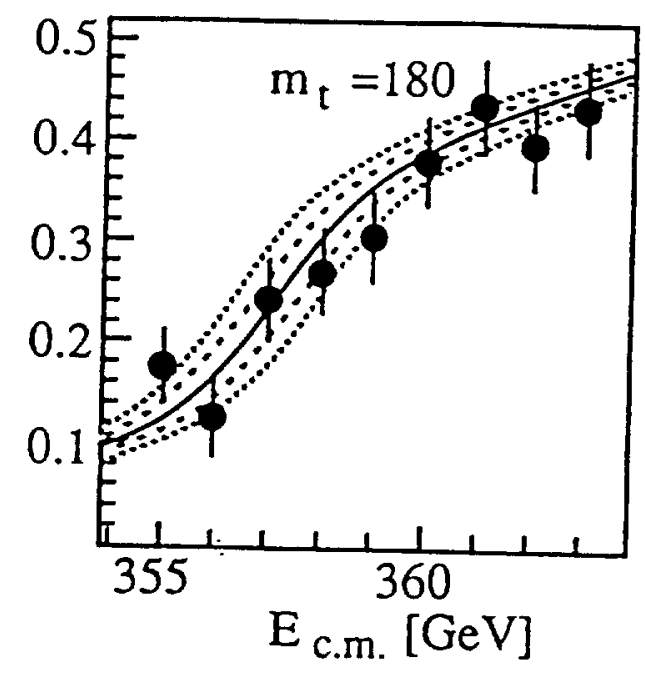

Abbildung 2: Produktion von top-Quarks in $e^{+} e^{-}$Kollisionen.

in $e^{+} e^{-}$Linearcollidern führen [6]. In den Präzisionsexperimenten, die an diesen Maschinen möglich sind, kann die top-Masse bis zu einer Genauigkeit von 200 $\mathrm{MeV}$ bestimmt werden [Abb. 2]; dies entspricht einer Verbesserung von mehr als einer Größenordnung im Vergleich zum LHC. Der Schlüssel zu dieser hohen Genauigkeit ist der schnelle Anstieg der Produktionsrate direkt oberhalb der Teilchenschwelle. Anomale magnetische Dipolmomente des top-Quarks wie auch mögliche CP-verletzende elektrische Dipolmomente können mit hoher Empfindlichkeit nachgewiesen werden. Desgleichen kann die Links-Chiralität der Zerfallsströme etabliert werden. In $e^{+} e^{-}$Linearcollidern läßt sich somit das physikalische Profil der topQuarks mit hoher Präzision zeichnen.

\section{(b) CP-VERLETZUNG}

Naturgesetze sind nicht symmetrisch beim gleichzeitigen Übergang von Teilchen zu Antiteilchen (C) und der Spiegelung des Raumes (P). Dieses Faktum ist im Komplex der $K, \bar{K}$ Mesonen entdeckt worden [7]. Die Verletzung dieser Symmetrie ist nicht allein von naturphilosophischem Interesse, sondern auch eine der notwendigen Bedingungen für die Existenz von Materie im Kosmos.

Im Rahmen des Standardmodells wird die Verletzung der CP-Symmetrie dadurch erklärt, daß sie bei der Wechselwirkung der fundamentalen Teilchen mit dem Higgs-Feld, das für die Erzeugung der Teilchenmassen verantwortlich ist, nicht respektiert wird. Aufgrund der Phasenfreiheit in den Wellenfunktionen der Quantenmechanik führt dies zu beobachtbaren Effekten, wenn die Anzahl der Familien nicht kleiner als drei ist [8]. In diesem Falle können schwache Übergänge zwischen Quarks unterschiedlicher Familien durch manifest komplexe, CP-verletzende Amplituden beschrieben werden.

Die bisherigen Analysen von Zerfallsexperimenten schränken die Übergangsamplituden so stark ein, daß eine scharfe Vorhersage für CP-verletzende Effekte im 
System der $B, \bar{B}$ Mesonen, die aus $b$-Quarks aufgebaut sind, möglich ist. Falls das CP Szenario des Standardmodells zutrifft, sollte das exponentielle Zerfallsgesetz von $B$ und $\bar{B}$-Strahlen durch eine Sinus-Modulation modifiziert werden. Dies wird in den Zerfällen $B, \bar{B} \rightarrow J / \psi+K$ nachweisbar sein. Experimente sind an asymmetrischen $e^{+} e^{-}$Collidern und am HERA-Protonenstrahl bei DESY in Vorbereitung. Sie werden nicht nur eine grundsätzliche Frage des Standardmodells klären, sondern zum ersten Mal Licht auf die Verletzung eines grundlegenden physikalischen Symmetriekonzeptes außerhalb des eingeschränkten $K, \bar{K}$ Komplexes werfen.

\section{(c) NEUTRINO-PHYSIK}

Es ist nicht unwahrscheinlich, daß Neutrinos eine kleine nicht-verschwindende Masse besitzen. Mit dieser Frage ist das Problem gekoppelt, ob neben den links-händigen Neutrinos der schwachen Wechselwirkung auch schwere inerte Neutrinos mit rechtshändigen Komponenten existieren. Solche Zustände können zu einer eleganten Lösung der Frage nach endlichen, aber sehr kleinen Massen der Standard-Neutrinos führen. Ebenso wie die Kopplung eines schnell mit einem langsam schwingenden Pendel zu einer extrem langsamen Eigenschwingung führt, so kann die Kopplung von leichten Neutrinos an schwere (Majorana-) Neutrinos zu sehr kleinen StandardNeutrinomassen führen. Die Massen von $\tau-, \mu$ - und $e$-Neutrinos würden in einem solchen Fall Werte von $10,10^{-3}$ und $10^{-7} \mathrm{eV}$ annehmen können.

Solch kleine Werte können dadurch gemessen werden, daß sich Oszillationen zwischen Neutrinos unterschiedlicher Familien in makroskopischen Zeiten über große Flugabstände ausbilden. In der Tat läßt sich das Defizit von solaren $e$-Neutrinos, welche die Erde aus der Wasserstoffusion der Sonne erreichen, aufgrund von Oszillationen in $\mu$-Neutrinos deuten. Dies impliziert Oszillation von $\mu$-in $\tau$-Neutrinos, die in Laborexperimenten in nächster Zeit nachgewiesen werden könnten. Quantitative Analysen [Abb. 3] sind verträglich mit der kosmologischen Schranke von etwa 10 $\mathrm{eV}$ an die Neutrinomassen, abgeleitet aus der Annahme, daß Neutrinos die heiße Komponente der dunklen Materie im Kosmos bilden.

\subsection{Die Fundamentalen Kräfte}

Zwischen Quarks und Leptonen wirken vier fundamentale Kräfte: Die elektromagnetische, die schwache und die starke Kraft können quantenfeldtheoretisch formuliert werden, wohingegen die Gravitationskraft lediglich ad hoc als klassisches Feld beigefügt wird. Die ersten drei Kräfte sind von gleicher Struktur. Sie werden durch Austausch von Spin-1 Teilchen erzeugt und besitzen eine gemeinsame eichtheoretische Basis. Das Gravitationsfeld trägt im Gegensatz dazu Spin-2, so daß die Gravitationskraft von gänzlich anderer Natur als die drei vorgenannten Kräfte ist.

Im Rahmen des Standardmodells ist es gelungen, die elektromagnetische und die schwache Kraft in einer einheitlichen elektroschwachen Wechselwirkung zusammenzufassen [1]. Die typische Energieskala der elektroschwachen Wechselwirkung liegt in der Größenordnung von $10^{2} \mathrm{GeV}$. [Die strukturelle Verwandtschaft der star- 


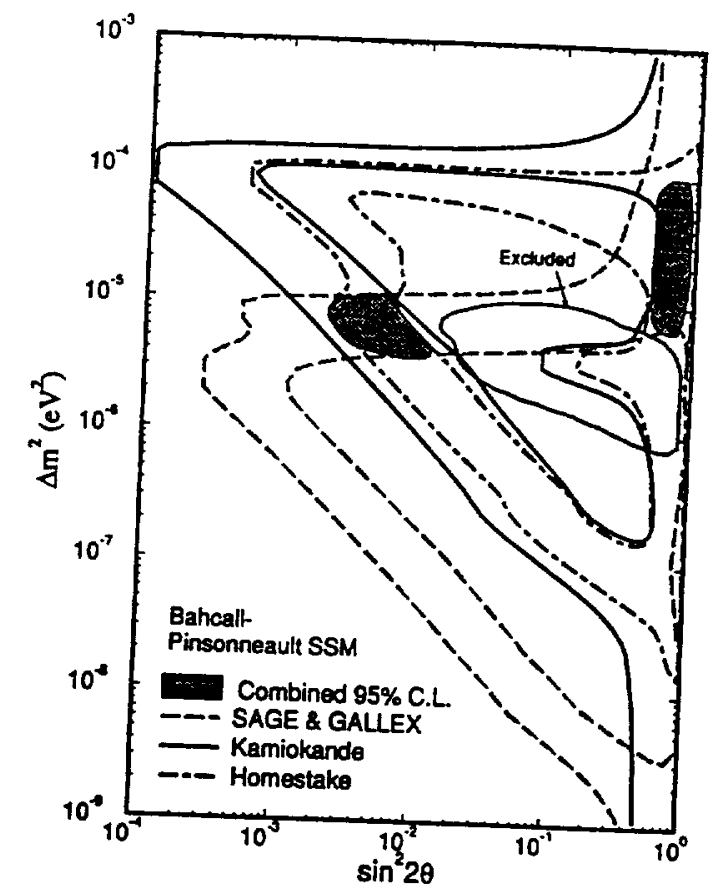

Abbildung 3: Abschätzung von Neutrinomassen aus der Oszillationsfrequenz von Sonnenneutrinos; $\Delta m$ kann mit der $\mu$-Neutrinomasse identifiziert werden. Ref. [9].

ken Kraft läßt vermuten, daß es eine große Vereinigung dieser drei Kräfte gibt; die zugehörige Skala wird in diesem Fall jedoch bei etwa $10^{16} \mathrm{GeV}$ liegen.]

\section{(a) ELEKTROSCHWACHE WECHSELWIRKUNGEN}

Die Quantenelektrodynamik als Teil der elektroschwachen Wechselwirkungen ist der Prototyp aller Eichfeldtheorien. Die elektromagnetische Kraft zwischen geladenen Teilchen wird durch den Austausch von Photonen erzeugt. Die Form der Wechselwirkung wird eindeutig festgelegt durch das Prinzip der abel'schen $U(1)_{E M}$ Eichinvarianz [10] und die Forderung, daß störungstheoretisch bestimmte Amplituden bis Wellenfunktionen geladener Teilchen in der sind. Die Eichinvarianz besagt, daß die Raum und Zeit abhängigen Phasen verser Quantenmechanik mit beliebigen, von sikalische Observable ändern; diese versehen werden können, ohne daß sich phyVektorfeldern. Die Unitaritätsforderung legt erzwingt die Einführung von Spin-1 Kopplung fest.

Die Quantenelektrodynamik ist eine Theorie von "astronomischer" Genauigkeit. Sie ist experimentell bis zu einer Präzision von $10^{-7}$ getestet worden und stellt damit die best-bestätigte Theorie in der Physik dar.

Die schwache Wechselwirkung ist im $\beta$-Zerfall der Kerne entdeckt worden. Im Zerfall eines Neutrons in ein Proton und ein Paar von Elektron und e-Antineutrino wird das Leptonenpaar mittels eines virtuellen geladenen $W$-Bosons an die Nukleonen (Quarks) gekoppelt. Der Zerfallsprozess wird unterdrückt durch die hohe Masse 
des $W$-Bosons, die bei $81 \mathrm{GeV}$ liegt, so daß die Übergangsamplitude klein ist und die Lebensdauer des Neutrons sehr lang wird.

Da die $W$-Bosonen Spin-1 besitzen müssen, um die Zerfallscharakteristiken des Neutrons richtig zu beschreiben, liegt es nahe, in Analogie zur Elektrodynanik die schwache Wechselwirkung eichfeldtheoretisch zu formulieren. Empirie und Unitarität führen dann notwendig zur Existenz von drei Eichbosonen, die untereinander trilinear und quattrolinear gekoppelt sind. Diese Form der Selbstwechselwirkung und ihre Stärke sind unter den vorgenannten Forderungen eindeutig festgelegt. Sie entsprechen einer nicht-abel'schen SU(2) Yang-Mills-Eichfeldtheorie [11].

Da die Elektrodynamik invariant ist unter Raumspiegelungen, die schwache Wechselwirkung diese Paritätsinvarianz jedoch verletzt, kann die neutrale Komponente des $S U(2)$ Vektorboson-Tripletts nicht mit dem Photon identifiziert werden. Die Symmetriegruppe muß daher zu $S U(2) \times U(1)$ erweitert werden. Zur $S U(2)$ gehören die geladenen Vektorbosonen $W^{ \pm}$und das neutrale Vektorboson $W^{3}$, zur $U(1)$ das neutrale Vektorboson $B$. Die beiden neutralen Vektorbosonen mischen miteinander, um die experimentell beobachteten Zustände des masselosen Photons und des massiven $Z$-Bosons zu bilden. Der Mischungswinkel wird im Parameter $\sin ^{2} \theta_{W}$ gemessen. Sein Wert liegt mit $\sim 0.23$ eine Größenordnung oberhalb des Wertes, den man von einer naiven störungstheoretischen Mischung erwartet hätte, so daß es gerechtfertigt ist, die elektroschwache Wechselwirkung als eine unifizierte Wechselwirkung anzusehen. Die Vorhersage der Mischung im Rahmen der großen Vereinigung aller Kräfte rechtfertigt diese physikalische Interpretation a posteriori.

Der experimentelle Durchbruch ist für die elektroschwache Theorie durch die Entdeckung der neutralen Stromreaktionen, die elastische Streuung von Neutrinos an Elektronen und Quarks, erzielt worden [12]. Der nächste große Schritt bestand im experimentellen Nachweis der massiven $W, Z$-Bosonen [13] und der Hochpräzisionsuntersuchung von $Z$-Bosonen [4]. Diese Gargamelle-, Proton-Antiproton Speicherring- und LEP-Experimente sind an anderer Stelle dieses Buches extensiv referiert worden [14].

Ein offenes Problem der elektroschwachen Wechselwirkungen ist die präzise Bestimmung der Selbstwechselwirkung von Vektorbosonen. Ihre Form und ihre Stärke werden durch die Eichfeldtheorie festgelegt. Diese können in Vorhersagen für die Werte der magnetischen Dipolmomente und elektrischen Quadrupolmomente der geladenen $W$-Bosonen transscribiert werden. Da eine Feldtheorie schwach gekoppelter Vektorbosonen, die bis zu asymptotischen Energien unitär ist, notwendig eine Eichfeldtheorie ist, müssen Abweichungen von diesen Vorhersagen klein sein, wenn das Standardmodell bis zu einem Bereich von $1 \mathrm{TeV}$ gültig sein soll. Genauigkeiten in der Größenordnung von $10^{-2}$ können am LHC unter Einschränkungen, definitiv jedoch erst an $e^{+} e^{-}$Linearcollidern mit einer Energie von $500 \mathrm{GeV}$ erzielt werden.

\section{(b) STARKE WECHSELWIRKUNG}

In frühen Zeiten der Teilchenphysik war versucht worden, ein selbst-konsistentes System für die stark-wechselwirkenden Hadronen, Baryonen und Mesonen, aufzubauen. 
Die dabei zugrundeliegenden Prinzipien waren Unitarität, Kausalität und der dynamische Ansatz der Dualität zwischen Resonanz- und Regge-Streuamplituden. Dieses nicht-lineare System war jedoch physikalisch nicht einschränkend genug, um zu einer eindeutigen Lösung für die Eigenschaften der Hadronen zu führen.

Das Rätsel der starken Wechselwirkung ist physikalisch von der Quantenchromodynamik, QCD, gelöst worden. Sie beruht auf zwei Hypothesen: (i) Hadronen sind aus Quarks aufgebaut [Ausnahmen von dieser Regel werden erst von der QCD selbst vorhergesagt]; (ii) Die Quarks tragen drei unterschiedliche Color-Ladungen, welche Quellen für gluonische Kraftfelder der starken Wechselwirkung bilden. Diese Hypothesen setzen den Rahmen für die Formulierung der starken Wechselwirkung
als nicht-abel'sche SU(3) Eichfeldtheorie [2].

Eine wichtige Eigenschaft der Quantenchromodynamik ist die asymptotische Freiheit [15]. Sie besagt, daß die Kopplung zwischen Quarks und Gluonen, und zwischen Gluonen untereinander, bei asymptotisch kleinen Abständen verschwindet. Umgekehrt wird sie sehr groß, wenn die Abstände im Bereich von einem Fermi [d.h. $10^{-13} \mathrm{~cm}$ ] liegen. Der Grund dafür liegt in Quantenfluktuationen, die jede Ladung umgeben und zur Polarisation des Vakuums führen. In der Quantenelektrodynamik bewirken kurzlebige Fermion-Antifermion Fluktuationen, daß das Vakuum notwendigerweise para-elektrisch wird und elektrische Ladungen folglich abgeschirmt werden. Der Wert einer elektrischen Ladung steigt darum an, wenn die Ladung bei kleinem Abstand gemessen wird. Umgekehrt in der Quantenchromodynamik. Para-colorelektrische Abschirmungseffekte von virtuellen Quark-Antiquarkund transversalen Gluon-Paaren werden überlagert von dia-colorelektrischen GluonFluktuationen, die von transversalen und Coulombischen Gluonen verursacht werden. Da letztere überwiegen, ist das QCD Vakuum insgesamt dia-colorelektrisch. Die starke Kopplung wird also kleiner mit verringertem Probeabstand und verschwindet asymptotisch gänzlich. Damit wird die starke Wechselwirkung bei hohen Energien störungstheoretischen Methoden der Quantenfeldtheorie zugänglich, während bei großen Abständen Methoden der Gitter-Eichfeldtheorien anwendbar werden. Hochenergie-Phänomene der starken Wechselwirkung wie auch statische Eigenschaften von Hadronen sind theoretisch beherrschbar geworden.

Die Quantenchromodynamik ist experimentell etabliert worden durch die Beobachtung von Gluon-Jets [16] im PETRA $e^{+} e^{-}$Speicherring [17] [siehe Abb. 4]. Die starke Beschleunigung der Colorladung bei der Produktion von Quarks in der $e^{+} e^{-}$ Annihilation führt zur Emission gluonischer Strahlung, die sich in Form eng kollimierter Hadron-Jets manifestiert [19]. Darüber hinaus sind wesentliche Elemente der QCD experimentell nachgewiesen worden [20]. Die Gluon-Selbstkopplung der nicht-abel'schen SU(3) Eichfeldtheorie konnte in den Winkelverteilungen von 4-Jet Endzuständen in Z-Zerfällen bei LEP aufgezeigt werden. Die Kombination von Messungen der starken Kopplung bei unterschiedlichen Energien folgt dem von der asymptotischen Freiheit vorhergesagten Abfall [Abb. 5].

Gitterrechnungen haben ein konsistentes Bild von statischen Hadroneigenschaften ergeben. Es ist gelungen, die Massen der wichtigsten Hadronen im Rahmen 


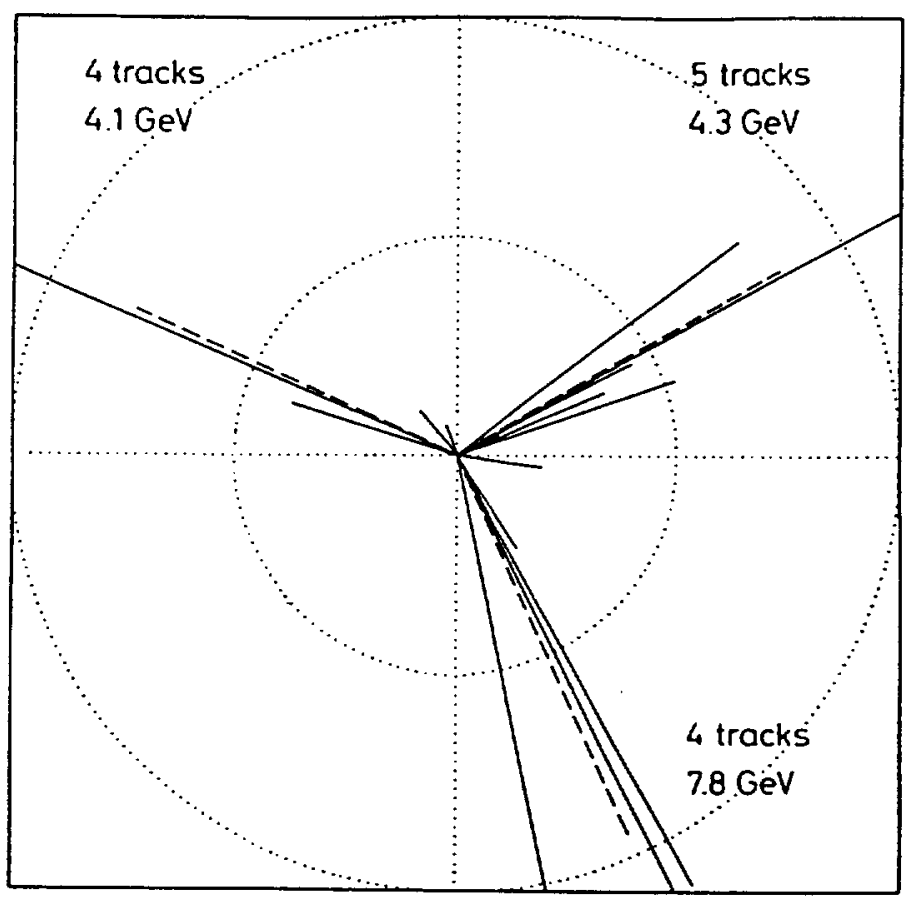

Abbildung 4: Gluonjet-Ereignis, beobachtet von der TASSO Collaboration in den Anfangsläufen von PETRA; Ref. [18].

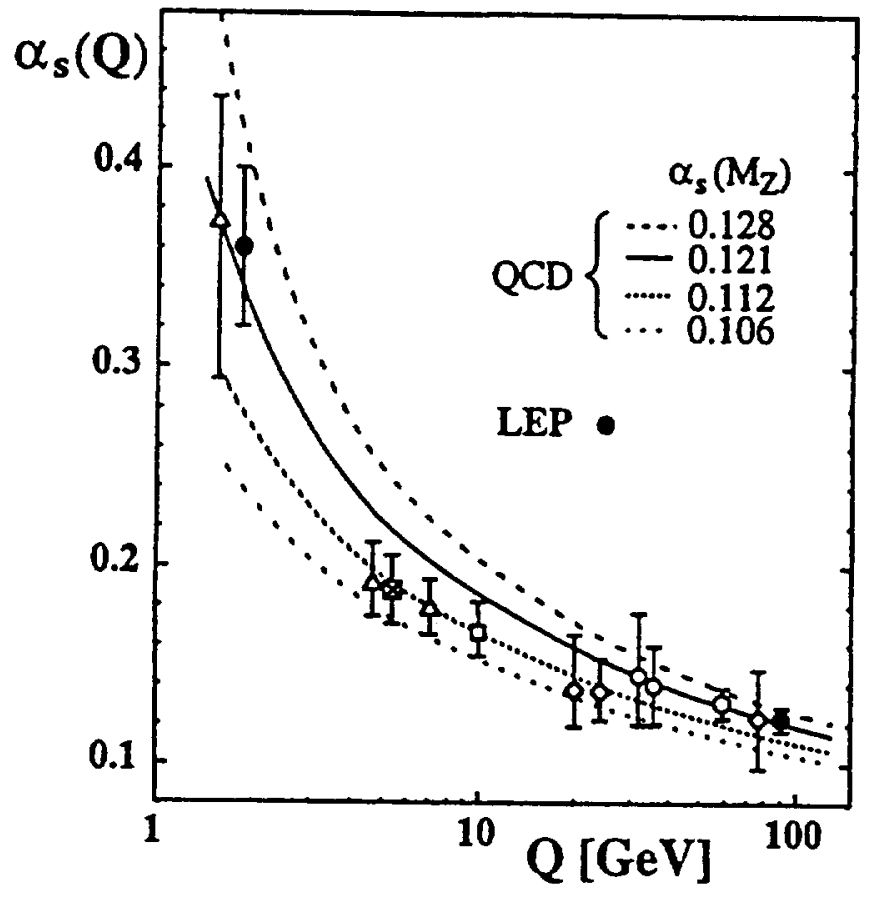

Abbildung 5: Asymptotische Freiheit: die Energieabhängigkeit der QCD Kopplung; Ref. [21]. 


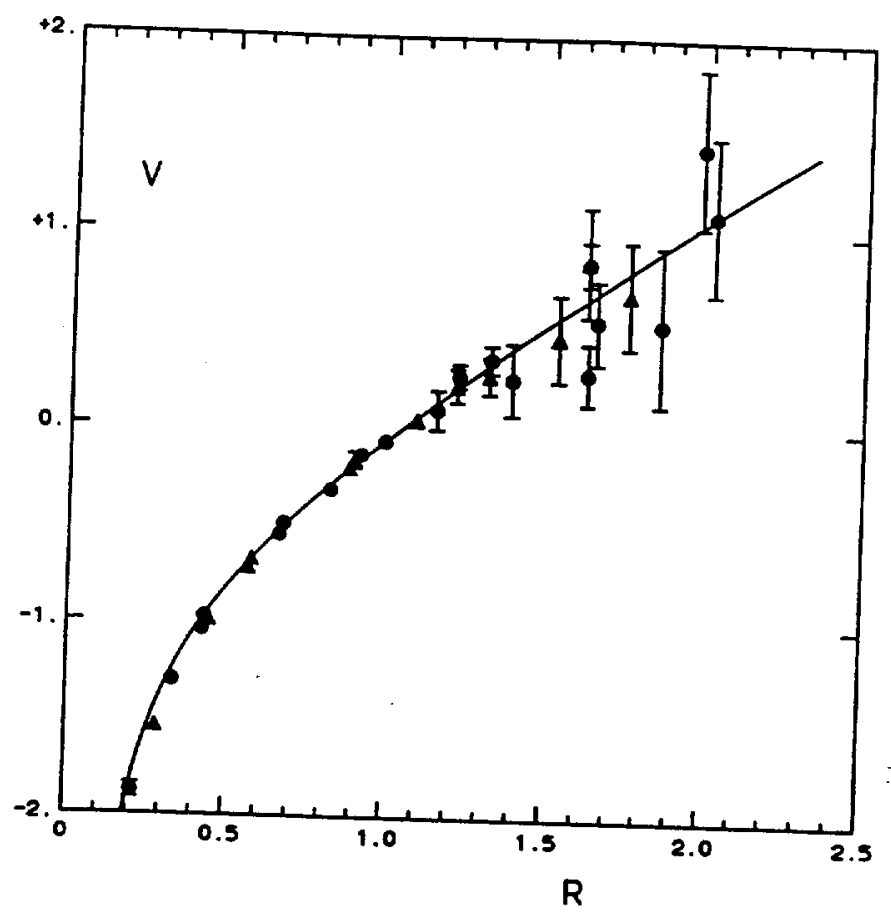

Abbildung 6: Das Potential zwischen schweren Quarks in der QCD; Ref. [23].

der QCD zu erklären [Tabelle 2]. Die Analyse des Interquark-Potentials [Abb. 6] legt ein lineares Verhalten bei großen Abständen nahe, was als Signal für das Confinement der Quarks interpretiert werden kann. Die QCD selbst verbietet es also,
daß Quarks als freie Teilchen auftreten können.

\begin{tabular}{||l|l|l||}
\hline Verhältnis & Gitter-QCD & Experiment \\
\hline \hline$m_{K_{*}} / m_{\rho}$ & $1.167 \pm 0.016$ & 1.164 \\
$m_{\Phi} / m_{\rho}$ & $1.333 \pm 0.032$ & 1.327 \\
$m_{N} / m_{\rho}$ & $1.219 \pm 0.105$ & 1.222 \\
$\Delta m / m_{\rho}$ & $1.930 \pm 0.073$ & 2.047 \\
$m_{\Delta} / m_{\rho}$ & $1.595 \pm 0.111$ & 1.604 \\
$m_{\Sigma^{*} / m_{\rho}}$ & $1.821 \pm 0.075$ & 1.803 \\
$m_{\Xi *} / m_{\rho}$ & $2.063 \pm 0.067$ & 1.996 \\
$m_{\Omega} / m_{\rho}$ & $2.298 \pm 0.098$ & 2.177 \\
& & \\
\hline
\end{tabular}

Tabelle 2: Hadronmassen; Ref. [22].

Weitere Vorhersagen der QCD, die Existenz von Hadronen, die nur aus Gluonen aber nicht aus Quarks aufgebaut sind, und das Deconfinement von Quarks bei 
hohen Temperaturen, harren noch der eindeutigen experimentellen Bestätigung.

\subsection{Higgs-Mechanismus}

Reine Eichtheorien sind bei schwacher Kopplung nur konsistent, wenn die assoziierten Vektorbosonen masselos sind. Massive Vektorbosonen besitzen longitudinale Polarisationsfreiheitsgrade, deren Wellenfunktionen mit der Energie ansteigen. Die elastische $S$-Wellen Streuamplitude longitudinal polarisierter Vektorbosonen, aufgebaut aus Austauschdiagrammen und der 4-Boson-Selbstkopplung, wächst quadratisch mit der Energie an, wohingegen die Unitarität eine asymptotisch konstante Amplitude verlangt. Die Theorie kann damit nur bis zu einer Energie von etwa 1 $\mathrm{TeV}$ gültig sein. Es gibt zwei Wege, dieses Problem zu lösen.

(i) Falls die Vektorbosonen bei Energien von $1 \mathrm{TeV}$ stark wechselwirkend werden, können die Streuamplituden so gedämpft werden, daß die Theorie asymptotisch unitär bleibt. Es ist jedoch bisher nicht gelungen, eine solch neue starke Wechselwirkung überzeugend zu formulieren. Technicolor-Theorien bieten mögliche Ansätze, sind jedoch in ihren einfachen Formen nicht mit den Präzisionsdaten der elektroschwachen Wechselwirkung konsistent.

(ii) Ein neuartiges Higgs-Teilchen wird eingeführt [24]. Der zusätzliche Austausch dieses Teilchens bei der elastischen Streuung von Vektorbosonen dämpft den quadratischen Anstieg in der Energie und führt so zu einer mit der Unitarität im Einklang stehenden Theorie. Dieses Zusammenspiel verlangt die Existenz eines skalaren Spin-0 Teilchens. Damit die Dämpfung der Streuamplituden eintritt, müssen seine Kopplungen an Quell-Felder mit den Massen der Quellen ansteigen; dies gilt ebenso für seine Selbstkopplung. Spin-0 und Kopplungen im Verhältnis der Massen sind also die beiden charakteristischen Eigenschaften von Higgs-Teilchen.

Theoretisch können diese Charakteristiken elegant re-interpretiert werden, indem die Eichtheorie mit spontaner Symmetriebrechung in skalaren Theorien verknüpft wird. Falls die Selbstwechselwirkung eines Skalarfeldes zu einem nichtverschwindenden Wert des Feldes im Grundzustand führt, können masselose Vektorbosonen und Fermionen mit diesem Untergrundfeld wechselwirken, so daß die Wechselwirkungsenergie zur Masse dieser Teilchen transmutieren kann. Der HiggsMechanismus zur Erzeugung von Teilchenmassen kann also physikalisch anschaulich als archimedischer Effekt in der Feldtheorie verstanden werden.

Da die Kopplungen durch den Higgs-Mechanismus bestimmt werden, sind alle Eigenschaften des Higgs-Teilchens a priori festgelegt, Zerfalls-Moden wie auch Produktionskanäle. Die einzige Ausnahme bildet die Higgs-Masse selbst, die mit der unbekannten Selbstwechselwirkung des Higgs-Feldes verknüpft ist. Jedoch existieren starke Einschränkungen an die möglichen Werte der Higgs-Masse [25, 26]. Obere Schranken können aus dem Anstieg der Higgs-Selbstkopplung mit der Energie abgeleitet werden. Falls die Higgs-Masse klein ist, kann das Standardmodell bis zur Planck-Skala fortgesetzt werden, bevor die Felder beginnen, stark miteinander zu wechselwirken. Eine kleine Higgs-Masse erlaubt es, den Wert des elektroschwachen 


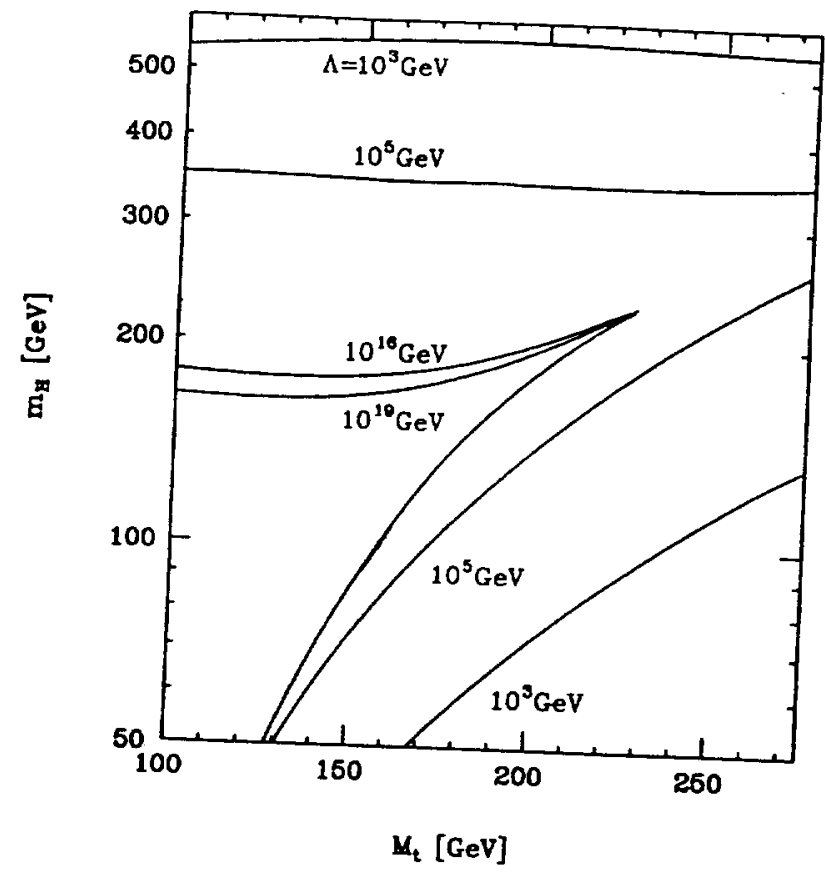

Abbildung 7: top-Quark und Higgs-Masse im Standardmodell; ltzt Ref. [25].

Mischungswinkels störungstheoretisch aus dem Symmetriewert an der großen Vereinigungsskala zu berechnen. Ist dagegen die Higgs-Masse groß, im Bereich von 700 $\mathrm{GeV}$, so tritt die starke Wechselwirkung bereits in der Energieregion von $1 \mathrm{TeV}$ auf. Untere Schranken werden andererseits von der Stabilität des Vakuums gefordert. Mit wachsender top-Quark Masse verringert sich der Wert der Higgs-Selbstkopplung durch Strahlungskorrekturen. Für zu große top-Massen würde er indefinit negativ, so daß der Higgs-Grundzustand nicht mehr stabil wäre. Der erlaubte Bereich ist in Abb. 7 dargestellt. In diesem Scenario ist für eine top-Masse von $175 \mathrm{GeV}$ nur ein Bereich zwischen $130 \mathrm{GeV}$ und $180 \mathrm{GeV}$ für die Higgs-Masse möglich, falls die Felder schwach wechselwirkend bleiben bis zur Planck-Skala. Der intermediäre Higgs-Massenbereich ist damit physikalisch bevorzugt.

Virtuelle Higgs-Teilchen beeinflussen elektroschwache Observable durch Strahlungskorrekturen. Indessen ist die Abhängigkeit von der Higgs-Masse nur logarithmisch und daher schwach. Elektroschwache Präzisionsanalysen zeichnen den Bereich kleiner Higgs-Massen aus, können jedoch Massen bis in den Bereich von 700 $\mathrm{GeV}$ nicht ausschließen. Nichtsdestoweniger wird damit der Higgs-Mechanismus zur Erzeugung von Teilchenmassen zum ersten Mal experimentell direkt gestützt [4].

Die Suche von Higgs-Teilchen bei LEP1 hat zu einer unteren Schranke von $65 \mathrm{GeV}$ geführt. Die Suche kann bei LEP2 bis zu Massenwerten von $100 \mathrm{GeV}$
fortgesetzt werden.

Falls die Masse oberhalb von $100 \mathrm{GeV}$ liegt, wird die Suche an den Hadroncollidern Tevatron und LHC fortgesetzt werden. Am LHC wird der gesamte klassische Wertebereich des Standardmodells überdeckt werden [27]. Damit sollte die Existenz 


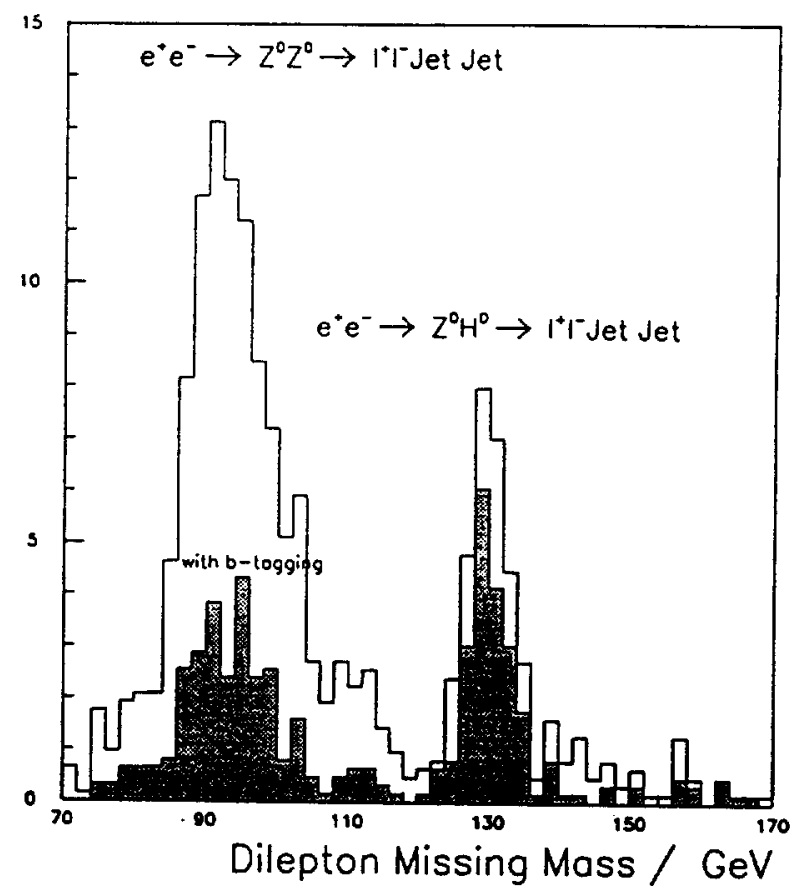

Abbildung 8: Signal für ein intermediäres Higgs-Boson in $e^{+} e^{-}$Linearcollidern Ref. [6].

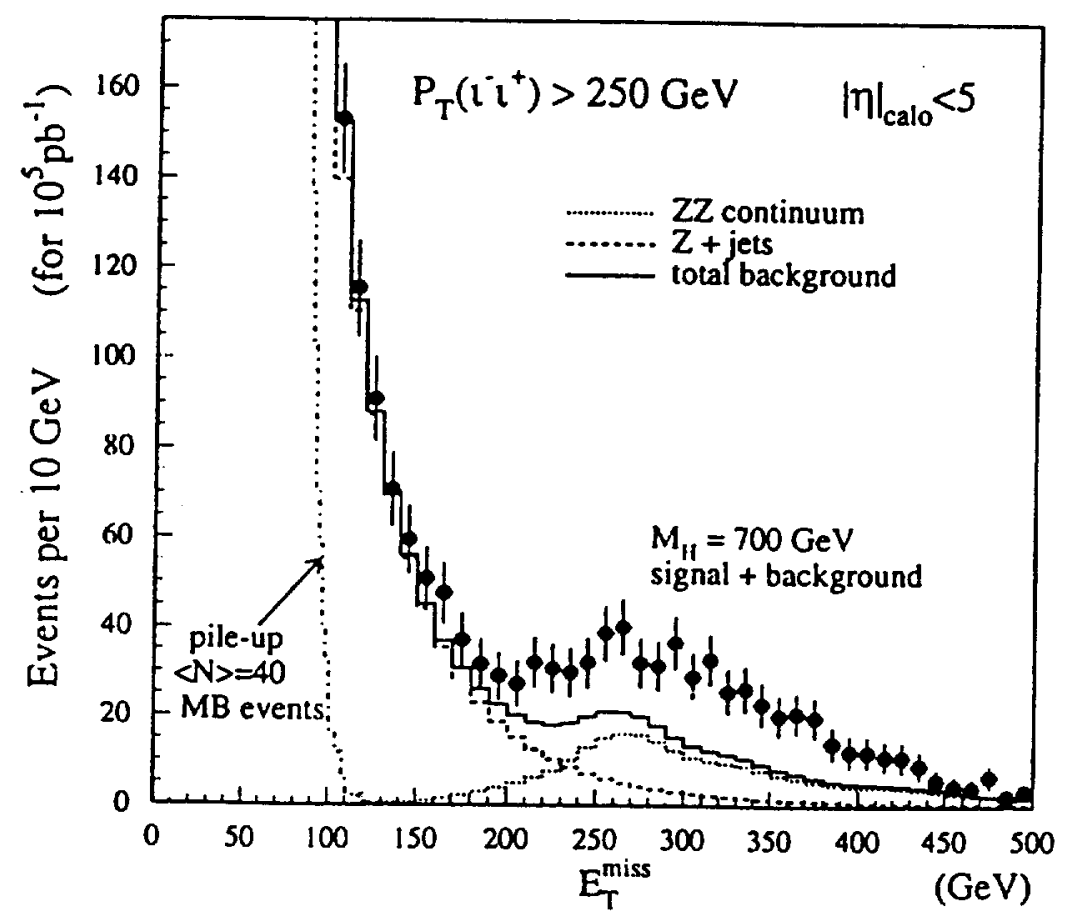

Abbildung 9: Signal für ein schweres Higgs-Boson im LHC Ref. [27]. 
des Higgs-Teilchens endgültig experimentell bestätigt oder widerlegt werden.

Die LHC Analysen sind im bevorzugten intermediären Bereich der HiggsMasse schwierig. Für diesen Bereich sind $e^{+} e^{-}$Linearcollider die idealen Maschinen [6]. Higgs-Teilchen können in diesen Anlagen praktisch untergrundfrei gefunden werden. Darüber hinaus gestatten es diese Anlagen, das Profil des Teilchens zu bestimmen. Aus der Produktionswinkelverteilung kann auf den Spin geschlossen werden. Die Messungen von Wirkungsquerschnitten für die Produktion und von Verzweigungsverhältnissen für Zerfälle bestimmen die Kopplungen an $W / Z$ Eichbosonen und Quarks/Leptonen. Bei Anlagen mit Energien von $1.5 \mathrm{TeV}$ kann sogar die Selbstkopplung der Higgs-Teilchen getestet werden, so daß wesentliche Elemente des Higgs-Potentials rekonstruiert werden können.

In der Kombination von LHC und $e^{+} e^{-}$Linearcollidern kann der gesamte Higgs-Bereich optimal überdeckt und die Natur des Teilchens erkannt werden. Mit diesem Tandem von Beschleunigern läßt sich der Higgs-Mechanismus experimentell etablieren, [Abb. 8] und [Abb. 9].

\section{Jenseits des Standardmodells}

Obwohl extrem erfolgreich, so muß das Standardmodell doch konstruktiver Kritik unterworfen werden. Ein wesentliches Element dieser Kritik ist die große Anzahl von freien Parametern, zu denen auch die beziehungslos nebeneinander stehenden Eichkopplungen gehören. Wenn das Standardmodell jedoch in Vereinigungstheorien mit sehr hohen Energieskalen eingebettet wird, in denen die Kopplungen miteinander identifiziert werden, so tritt ein Hierarchie-Problem für leichte Higgs-Massen auf: Strahlungskorrekturen treiben die Higgs-Masse in die Nähe der hohen Vereinigungsskala. Weitere Probleme werden offensichtlich durch die Notwendigkeit verursacht, die Gravitation quantentheoretisch in die Theorie einzubauen. Und letztlich muß es möglich sein, in einer Theorie der Materie die im Standardmodell inkorporierten Symmetrien selbst aus übergeordneten Prinzipien abzuleiten. Dies gilt für Eichsymmetrien wie auch diskrete Spiegelungssymmetrien und ihre Brechungsmechanismen.

Es ist nicht ausgeschlossen, daß Superstringtheorien Kandidaten für die Lösung eines solch ambitionierten Programms sind. Im gegenwärtigen Kontext sollen jedoch nur zwei sich gegenseitig bedingende Schritte in diese Richtung skizziert

\subsection{Grosse Vereinigungs-Symmetrie}

Die Symmetriegruppe des Standardmodells ist das direkte Produkt $S U(3) \times S U(2) \times$ $U(1)$ für die starke und elektroschwache Wechselwirkung. Dies ist eine Untergruppe der einfachen Gruppe $S U(5)$, die sich damit als übergeordnete Symmetriegruppe bei hohen Energien anbietet [28]. Diese Hypothese wird darüber hinaus von der Beobachtung gestützt, daß Leptonen und Quarks jeder Familie in ein Quintuplett 
und ein Dekuplett der $S U(5)$ eingeordnet werden können.

An der Vereinigungsskala ist der Wert des elektroschwachen Mischungswinkels, der das Verhältnis von schwacher zu elektromagnetischer Kopplung beschreibt, durch die Symmetriegruppe festgelegt zu $\sin ^{2} \theta_{W}^{s y m}=3 / 8$. Infolge von Vakuumpolarisationseffekten verringert er sich $\mathrm{zu} \sim 0.21$, wenn er störungstheoretisch in den Niederenergiebereich von $100 \mathrm{GeV}$ fortgesetzt wird [29]. Der resultierende Wert liegt zwar in der Nähe des experimentellen Meßwertes, verfehlt ihn jedoch bei genauer Analyse um etwa 0.02 Einheiten. Zwei Schlüsse bieten sich aus diesem Vergleich an. Einerseits wird die Grundidee gestützt, das Standardmodell von einer Energieskala von $100 \mathrm{GeV}$ bis zu einer Vereinigungsskala in der Nähe der Planck-Skala fortzusetzen; andererseits ist jedoch das Teilchenspektrum des Standardmodells, das die Änderung des elektroschwachen Mischungswinkels mit der Energie bestimmt, unvollständig und muß durch weitere Teilchen supplementiert werden. Dieser Schritt führt in natürlicher Weise zu einer supersymmetrischen Erweiterung des Standardmodells. In der Minimalform und verwandten Formen dieser Erweiterung gelingt die quantitative Deutung des elektroschwachen Mischungswinkels.

\subsection{Supersymmetrie}

Die Supersymmetrie [30] ist ein Symmetriekonzept, das Fermionen und Bosonen zueinander in Beziehung setzt. Multipletts, die dieser Symmetrie zugeordnet sind, vereinen beide Teilchenarten; sie bestehen also aus Teilchenpaaren, die sich im Spin um eine 1/2 Einheit unterscheiden. Die minimale supersymmetrische Erweiterung des Standardmodells umfaßt das in [Tabelle 3] aufgelistete Teilchenspektrum.

\begin{tabular}{||c|c|l|c||}
\hline Teilchen & Spin & SUSY-Teilchen & Spin \\
\hline \hline & & & \\
Lepton $l$ & $1 / 2$ & Slepton $\tilde{l}$ & 0 \\
Quark $q$ & $1 / 2$ & Squark $\tilde{q}$ & 0 \\
Gluon $g$ & 1 & Gluino $\tilde{g}$ & $1 / 2$ \\
$\gamma, W^{ \pm}, Z$ & 1 & Gaugino $\tilde{\gamma}, W^{ \pm}, \tilde{Z}$ & $1 / 2$ \\
Higgs $H$ & 0 & Higgsino $\tilde{H}$ & $1 / 2$ \\
\hline
\end{tabular}

Tabelle 3: Teilchenspektrum der minimalen supersymmetrischen Erweiterung des Standardmodells.

Jedem Lepton und Quark wird ein skalares Slepton und Squark zugeordnet. Die vektoriellen Eichbosonen werden mit Spin-1/2 Gauginos gepaart. Bei Respektierung der Supersymmetrie erfordert die Erzeugung von Massen für up- und down-Quarks eine Erweiterung des Higgs-Sektors, aus dem insgesamt fünf neutrale 
und geladene physikalische Higgs-Bosonen hervorgehen. Im fermionischen Sektor entsprechen ihnen acht neutrale und geladene Higgsinos.

Es gibt vielfältige physikalische Argumente für die Hypothese, daß die Supersymmetrie in der Natur realisiert ist, wenn auch in gebrochener Form mit unterschiedlichen Massen für die fermionischen und bosonischen Partner innerhalb eines Multipletts.

(a) Stabilisierung des Higgs-Sektors und Massen: Wenn das Standardmodell in eine große Vereinigungstheorie eingebettet wird, wie vom Wert des elektroschwachen Mischungswinkels nahegelegt, so werden Strahlungskorrekturen im allgemeinen zu einer Higgs-Masse knapp unterhalb der Vereinigungsskala führen. Wenn jedoch zu Bosonen zusätzlich Fermionen in das Spektrum eingeführt werden, so heben sich die großen Beiträge beider Teilchensorten zu den Strahlungskorrekturen gegenseitig auf [31], eine Folge des Pauli-Prinzips. Es ist also keine ad hoc Feinjustierung von Parametern um viele Größenordnungen nötig, um eine leichte Higgs-Masse in der Region von $100 \mathrm{GeV}$ zu stabilisieren. Durch die Supersymmetrie des Teilchenspektrums wird dies in natürlicher Weise erreicht. Um die Feinjustierung auch bei Brechung der Supersymmetrie zu vermeiden, können die Massen supersymmetrischer Teilchen nicht den Bereich von etwa $1 \mathrm{TeV}$ überschreiten; die leichtesten der color-neutralen Gauginos und Higgsinos sollten sogar Massen unterhalb von $200 \mathrm{GeV}$ besitzen.

(b) Supersymmetrische Grosse Vereinigungstheorie: Die zusätzlichen Teilchen supersymmetrischer Theorien, die das Spektrum des Standardmodells supplementieren, ändern die Vakuumpolarisation, so daß die Energieabhängigkeit der starken und elekroschwachen Kopplungen modifiziert wird. Im Rahmen der minimalen Supersymmetrie wird der Wert des elektroschwachen Mischungswinkels bei einer Energie von $100 \mathrm{GeV}$ zu $\sin ^{2} \theta_{W}=0.2335 \pm 0.0017$ vorausgesagt $[32,33]$. Diese Vorhersage ist in sehr guter Übereinstimmung mit dem Meßwert $\sin ^{2} \theta_{W}=$ $0.2315 \pm 0.0004$. Zwar beweist die Übereinstimmung nicht, daß die Supersymmetrie in der Natur realisiert ist - was nur durch die Beobachtung supersymmetrischer Teilchen geschehen kann - jedoch erhöht sie in starkem Maße die indirekte Evidenz für diese neuartige Symmetrie.

Mit der Supersymmetrie ist ein Mechanismus entdeckt worden, der es erlaubt, die Theorie von tiefen Energien im Bereich von $100 \mathrm{GeV}$ bis in die Nähe der PlanckSkala von $10^{19} \mathrm{GeV}$, bei der die Gravitation eingebunden werden muß, in konsistenter Weise fortzusetzen.

(c) Radiative Symmetriebrechung: Der Higgs-Mechanismus muß ins Standardmodell ad hoc zur Erzeugung der Massen fundamentaler Teilchen eingeführt werden. Bettet man hingegen die supersymmetrische Theorie in eine große Vereinigungstheorie ein, so kann der Higgs-Mechanismus in seiner Essenz theoretisch abgeleitet werden. Falls alle skalaren Teilchen an der Vereinigungsskala eine gemeinsame Masse besitzen, so ändern Strahlungskorrekturen bei Fortsetzung der Theorie zu tiefen Energien die Massenparameter der Higgs-Felder in der Weise ab, daß die Selbstwechselwirkung zur spontanen Symmetriebrechung im $S U(2) \times U(1)$ Sektor führt, die Color $S U(3)$ Symmetrie und die elektromagnetische $U(1)_{E M}$ Symmetrie je- 
doch ungebrochen bleiben [34]. Die treibende Kraft ist dabei das top-Quark: Dieser radiative Mechanismus für die Brechung der $S U(2) \times U(1)$ Symmetrie bei gleichzeitiger Respektierung der Color und elektromagnetischen Eichsymmetrien kann nur realisiert werden, wenn die top-Quark Masse in einem Bereich von 150 bis $200 \mathrm{GeV}$ liegt - also genau dort, wo sie experimentell gefunden wurde. Damit ermöglicht die Supersymmetrie eine physikalisch tiefgehende Deutung des Higgs-Mechanismus.

Die Suche nach supersymmetrischen Teilchen gehört zu den wichtigsten experimentellen Aufgaben an den gegenwärtigen und zukünftigen Collider-Anlagen $[6,27]$. Je nach Teilchensorte sind Hadron- oder $e^{+} e^{-}$Collider die geeigneten Maschinen, diese Teilchen aufzuspüren:

- Higgs-Teilchen: Im Quintuplett der neutralen und geladenen Higgs-Bosonen besitzt das leichteste Teilchen eine Masse unterhalb von $150 \mathrm{GeV}$, bei kleinem Wert eines Mischungsparameters $\tan \beta$ sogar unterhalb von $100 \mathrm{GeV}$. Dieses Higgs-Teilchen könnte also bei LEP2 entdeckt werden. Wenn nicht dort, so wird das Teilchen mit Sicherheit an einem $e^{+} e^{-}$Linearcollider erzeugt werden können. Im LHC kann der supersymmetrische Higgs-Sektor nur überdeckt werden bei einer Überlagerung sehr vieler unterschiedlicher Kanäle.

- Neutralinos/Charginos und Sleptonen: Die ersteren sind Mischungen colorneutraler Gauginos und Higgsinos. Sie sind vermutlich die leichtesten der supersymmetrischen Teilchen und können in $e^{+} e^{-}$Collidern sehr effektiv gesucht und, falls gefunden, auch untersucht werden. In Hadroncollidern können sie sich in komplizierten Kaskaden-Zerfällen durch Multilepton-Endzustände bemerkbar machen. Sleptonen werden in $e^{+} e^{-}$Collidern in ähnlich einfacher Weise erzeugt und experimentell nachgewiesen wie Leptonen selbst; in Hadroncollidern ist die Produktionsrate für Sleptonen nur am untersten Ende des Massenspektrums groß genug, um die Entdeckung dieser Teilchen zu ermöglichen.

- Squarks und Gluinos: Diese Teilchen sind vermutlich die schwersten Partikel im supersymmetrischen Spektrum. In Hadroncollidern werden sie in großer Anzahl erzeugt, sobald die Schwellenenergie erreicht wird. Am Tevatron werden sie zugänglich sein, falls ihre Massen den Wert von 300 bis $400 \mathrm{GeV}$ nicht überschreiten; am LHC werden sie mit Massen bis zu $2 \mathrm{TeV}$ entdeckt werden können. Ihre Eigenschaften können jedoch nur grob in diesen Anlagen bestimmt werden.

Mit der Kombination von Hadron- und $e^{+} e^{-}$Collidern wird eine ideale Konstellation für die Supersymmetrie realisiert. Die Hadroncollider, Tevatron und LHC, können Squarks und Gluinos über den gesamten kanonischen Massenbereich verfolgen, ebenso wie LEP2 und $e^{+} e^{-}$Linearcollider die color-neutralen Teilchen, Neutralinos/Charginos und Sleptonen. Darüber hinaus lassen sich die Eigenschaften der Teilchen in $e^{+} e^{-}$Anlagen präzise bestimmen. Dies wird es erlauben, Rückschlüsse auf unterliegende Vereinigungstheorien zu ziehen, so daß ein experimentelles Fenster zu Energiebereichen bis in die Nähe der Planck-Skala geöffnet werden kann. 


\section{Summa}

Mit dem Standardmodell ist eine Theorie formuliert worden, welche die Gesetzmäßigkeiten des Mikrokosmos in prägnanter Form zusammenfaßt. Wesentliche Elemente dieser Theorie sind experimentell etabliert worden: Fermionspektrum und Kraftfelder. Weitere grundlegende Komponenten, Eichcharakter der elektroschwachen Wechselwirkung und CP-Verletzung, harren detaillierter experimenteller Untersuchung. Der Higgs-Mechanismus zur Erzeugung von Teilchenmassen ist bisher noch nicht etabliert, jedoch ergeben experimentelle Resultate erste Evidenz für die Realisierung dieses Mechanismus in der Natur.

Viele Züge des Standardmodells weisen auf die Einbettung des Modells in eine übergeordnete Theorie hin. Ein überzeugendes Konzept stellt die Supersymmetrie dar, realisiert in einem Massenbereich unterhalb von $1 \mathrm{TeV}$. Eine solche Theorie kann konsistent fortgesetzt werden bis in den Energiebereich nahe der Planck-Skala, in dem die elektroschwache und die starke Kraft $\mathrm{zu}$ einer einheitlichen Wechselwirkung zusammengefaßt werden können. Die Supersymmetrie erlaubt es also, eine Brücke vom Niederenergiebereich zur Planck'schen Energieregion zu schlagen, in der die Gravitation, die vierte der Wechselwirkungen, quantentheoretisch eingebunden werden muß.

Die gegenwärtigen Beschleunigeranlagen sowie zukünftige Collider, der Protonencollider LHC und $e^{+} e^{-}$Linearcollider, können in Verfolgung dieses physikalischen Programms Meilensteine auf dem Weg zu einer umfassenden Theorie der Materie setzen.

\section{Danksagung}

Herrn Prof. Dr. S. Bethke und Herrn Dr. D. Rein möchte ich sehr herzlich für die Einladung zum Physikalischen Kolloquium aus Anlaß der 125 Jahrfeier der RWTH Aachen danken.

\section{Literatur}

[1] S.L. Glashow, Nucl. Phys. 22 (1961) 579;

S. Weinberg, Phys. Rev. Lett. 19 (1967) 1264;

A. Salam, in Elementary Particle Theory, ed. N. Svartholm (Stockholm 1968).

[2] H. Fritzsch und M. Gell-Mann, Proc. XVI Int. Conf. on High Energy Physics, eds. J.D. Jackson et al. (Chicago 1972). 
[3] G. Köpp, D. Schaile, M. Spira und P.M. Zerwas, Z. Phys. C65 (1995) 545;

S. Aid et al. [H1], Phys. Lett. B353 (1995) 578;

F. Abe et al. [CDF], FERMILAB-PUB-96-020-E.

[4] D. Schaile, Proc. XXVII Int. Conf. on High Energy Physics, eds. P. Bussey und I. Knowles, (Glasgow 1994).

[5] F. Abe et al. [CDF], Phys. Rev. Lett. 73 (1994) 225 und 74 (1995) 2626;

S. Abachi et al. [D0], Phys. Rev. Lett. 74 (1995) 2632.

[6] Proceedings, $e^{+} e^{-}$Collisions at $500 \mathrm{GeV}$ : The Physics Potential, ed. P.M. Zerwas, DESY 92/96-123A/D.

[7] J.H. Christenson, J.W. Cronin, V.L. Fitch und R. Turlay, Phys. Rev. Lett. 13 (1964) 138.

[8] N. Cabibbo, Phys. Rev. Lett. 10 (1963) 531;

M. Kobayashi und T. Maskawa, Prog. Theor. Phys. 49 (1973) 652.

[9] P. Langacker, Proceedings, Beyond the Standard Model IV, (Lake Tahoe 1994).

[10] H. Weyl, Z. Phys. 56 (1929) 330.

[11] C.N. Yang und R.L. Mills, Phys. Rev. 96 (1954) 191.

[12] F.J. Hasert et al. [GARGAMELLE], Phys. Lett. 46B (1973) 121 und 138.

[13] G. Arnison et al. [UA1], Phys. Lett. B122 (1983) 103 und 126 (1983) 398;

M. Banner et al. [UA2], Phys. Lett. B122 (1983) 476 und 129 (1983) 130.

[14] D. Haidt und G. Herten, an späterer Stelle dieses Buches.

[15] D.J. Gross und F. Wilczek, Phys. Rev. Lett. 30 (1973) 1343;

H.D. Politzer, Phys. Rev. Lett. 30 (1973) 1346.

[16] J. Ellis, M.K. Gaillard und G.G. Ross, Nucl. Phys. B111 (1976) 253.

[17] R. Brandelik et al. [TASSO], Phys. Lett. B86 (1979) 243;

D.P. Barber et al. [MARK J], Phys. Rev. Lett. 43 (1979) 830;

Ch. Berger et al. [PLUTO], Phys. Lett. B86 (1979) 418;

W. Bartel et al. [JADE], Phys. Lett. B91 (1980) 142.

[18] B.H. Wiik, Proceedings, Neutrino Conference (Bergen 1979).

[19] P. Hoyer, P. Osland, H.G. Sander, T.F. Walsh und P.M. Zerwas, Nucl. Phys. B161 (1979) 349.

[20] H.G. Sander, an späterer Stelle dieses Buches.

[21] S. Bethke, Nucl. Phys. B (Proc. Suppl.) 39B,C (1995) 198. 
[22] F. Butler, H. Chen, J. Sexton, A. Vaccarino und D. Weingarten, Nucl. Phys. B430 (1994) 179.

[23] K.D. Born, E. Laermann, T.F. Walsh und P.M. Zerwas, Phys. Lett. B329 (1994) 332.

[24] P.W. Higgs, Phys. Rev. Lett. 12 (1964) 132 und 13 (1964) 50s;

F. Englert und R. Brout, Phys. Rev. Lett. 13 (1964) 321;

G.S. Guralnik, C.R. Hagen und T. Kibble, Phys. Rev. Lett. 13 (1964) 585.

[25] N. Cabibbo, L. Maiani, G. Parisi und R. Petronzio, Nucl. Phys. B158 (1979) 295;

M. Lindner, M. Sher und H.W. Zaglauer, Phys. Lett. B228 (1989) 139;

J.A. Casas, J.R. Espinosa und M. Quiros, Phys. Lett. B342 (1995) 171.

[26] A. Hasenfratz, K. Jansen, J. Jersak, C.B. Lang, T. Neuhaus und H. Yoneyama, Nucl. Phys. B317 (1989) 81.

[27] CMS Collaboration, Techn. Proposal CERN LHCC/94-38;

ATLAS Collaboration, Techn. Proposal CERN LHCC/94-43.

[28] H. Georgi und S.L. Glashow, Phys. Rev. Lett. 32 (1974) 438.

[29] H. Georgi, H.R. Quinn und S. Weinberg, Phys. Rev. Lett. 33 (1974) 451.

[30] J. Wess und B. Zumino, Nucl. Phys. B70 (1974) 39.

[31] E. Witten, Nucl. Phys. B188 (1981) 513.

[32] L.E. Ibanez und G.G. Ross, Phys. Lett. B105 (1981) 439;

S. Dimopoulos, S. Raby und F. Wilczek, Phys. Rev. D24 (1981) 1681.

[33] P. Langacker und M.-X. Luo, Phys. Rev. D44 (1991) 817;

U. Amaldi, W. de Boer und H. Fürstenau, Phys. Lett. B260 (1991) 447;

J. Ellis, S. Kelley und D.V. Nanopoulos, Phys. Lett. B260 (1991) 131.

[34] L.E. Ibanez und G.G. Ross, Phys. Lett. B110 (1982) 215. 